\title{
NOTEN ZUM TEXT VON OVIDS TRISTIEN
}

JOHANNES SCHWIND

Universität Trier

This article discusses the textual constitution of the following passages from Ovid's Tristia in J. B. Hall's editio Teubneriana (1995): I 3.99; 5.81; 2.6sq., 53, 324, 540; III 4.24; 11.41; IV 1.7;

$2.36 ; 6.26,34 ; 9.7 ; \mathrm{V} 6.1-3 ; 13.30$.

Ihren Ausgangspunkt nehmen die folgenden Zeilen von der jüngsten kritischen Ausgabe von Ovids Tristien, der Teubneriana von J. B. Hall (Stuttgart Leipzig 1995), deren Text in einem bei Klassikereditionen ungewohnten Ausmaß von dem ihrer Vorgängerinnen abweicht ${ }^{1}$. Unter dem Motto «Even if there be nothing in fact to heal, a little probing here and there may at least have the effect of drawing attention to something unusual and worthy of a word or two of comment» (Euphrosyne 20, 1992, S. 131) hatte Hall bereits zuvor in einer Reihe von Aufsätzen zahlreiche, z.T. tief in die Überlieferung eingreifende Konjekturen vorgeschlagen ${ }^{2}$. Sie alle und darüber hinaus noch viele weitere, insgesamt mehr als 150, finden Eingang in seine Tristienausgabe. Eine solch hohe Zahl von editorischen Eingriffen würde auch ohne das oben zitierte Motto des Herausgebers mißtrauisch stimmen. Ist es denn überhaupt

So von der Oxoniensis Owens an über 600 Stellen. Vgl. die Rezensionen von B. W. Häuptli, $M H$ 53, 1996, S. 325f., und J. Schwind, CR 47, 1997, S. 300ff.

2 J.B. Hall: «Notes on various passages in Ovid's Tristia», Euphrosyne 16, 1988, Ss. 125138; «Problems in Ovid's Tristia», PCPhS Suppl. 15, 1989, Ss. 20-38; «More Notes on Ovid's Tristia», Euphrosyne 18, 1990, S. 85-98; «Ovid, Tristia 2.77-80 and 5.11.25-28, LCM 16.3, 1991, S. 37f.; «Seven Notes on Ovid's Tristia», LCM 16.6, 1991, S. 83f.; «Additional Notes on Ovid's Tristia», Euphrosyne 20, 1992, Ss. 131-148. 
denkbar, daß ein seit Jahrhunderten philologisch traktierter Klassikertext von 3.500 Versen Umfang an 150 Stellen einer Heilung bedarf? Im folgenden sollen einige der Textgestaltungen Halls erörtert werden. Dabei wird in Abschnitt I zu zeigen sein, daß sich der überlieferte Text, wenn Sinn und Intention nur richtig erfaßt werden, vielfach als besser erweist als der konjektural hergestellte. Der Abschnitt II ist Textstellen gewidmet, an denen Hall Änderungen unter Berufung auf Ovids Stilwollen vornimmt. Aus generellen methodologischen Erwägungen wie aus jeweils verschiedenen sachlichen Gründen werden sich auch hier einige Konjekturen als nicht akzeptabel herausstellen. Der Verfasser hofft, mit seinen Ausführungen zugleich zu einem tieferen Verständnis der einen oder anderen Stelle von Ovids Tristien beitragen zu können.

\section{I}

II 323f. denique cum meritis impleueris omnia, Caesar, pars mihi de meritis (trad. multis) una canenda fuit.

Die Konjektur Halls resultiert offenbar aus einem Fehlverständnis der Aussageabsicht des Dichters. Bei de multis ist gedanklich nicht meritis aus dem vorhergehenden Vers zu ergänzen ${ }^{3}$, sondern partibus aus pars in der unmittelbaren Nähe: Ovid gesteht ein, daß er wenigstens einen einzigen von den vielen Bereichen, in denen Augustus Großtaten vollbrachte, als Dichter hätte besingen müssen. Die Änderung des überlieferten Textes ist nicht nur unnötig, sie bringt sogar eine Verschlechterung, insofern sie die von Ovid geliebte antithetische Juxtaposition von multi und unus $^{4}$ zugunsten einer pointenlosen Wiederholung von meritis beseitigt.

II 539f. nos quoque iam pridem scripto peccauimus uno: supplicium patitur non noua cura (trad. culpa) nouum.

Das überlieferte Nomen culpa ist nicht nur gut verständlich, sondern aus Gründen der Gedankenführung und ihrer stilistischen Gestaltung unabdingbar.

\footnotetext{
3 Von dieser Annahme geht Hall aus: vgl. Euphrosyne 22, 1992, S. 138.

4 Der vorliegenden Stelle am nächsten kommen Trist. I 3.16 qui modo de multis unus et alter erant und Remed. 682 iam facito e multis una sit illa tibi, wo die beiden Opposita ebenfalls durch die Mittelzäsur des Pentameters getrennt werden. Vgl. auch Trist. II 376 dum uir abest, multis una petita procis und, ohne unmittelbare Juxtaposition, Ars I 344 uix erit e multis, quae neget, una, tibi, III 422 quem trahat, e multis forsitan unus erit, Remed. 463, Pont. III 9.5 o! quam de multis uitium reprehenditur unum.
} 
Ovid beschwert sich, daß andere Dichter für ihre lasziven Jugendwerke nicht zur Rechenschaft gezogen worden seien. Gewiß habe er sich durch das Verfassen der Ars amatoria vergangen (v. 539), doch sei seine Bestrafung etwas noch nie Dagewesenes (nouиm), insofern sie ein uraltes (non noua) Vergehen ahnde (v. 540). Mit non noua culpa nimmt der Dichter einerseits pridem peccauimus aus dem vorhergehenden Vers wieder auf, zum andern produziert er durch die Antithese der in gewisser Weise komplementären Begriffe culpa und supplicium, die beide mit dem - freilich semantisch verschieden gelagerten - Attribut nouus ausgestattet sind, in spielerischer Weise ein "Paradoxon": die non noua culpa findet ein nouum supplicium. Es besteht kein Bedarf für einen konjekturalen Eingriff.

Warum scheiterte Icarus, nicht aber Dädalus?

III 4,23f. nempe quod hic alte, demissius ille uolabat; nam pinnas ambo non tenuere pares (trad. non habuere suas).

Die hier vorliegende, besondere und gerade von Ovid geliebte Verwendung des Possessivpronomens hat der Herausgeber nicht erkannt, wenn er zur Stelle schreibt: «and if they did not have 'their own' wings, whose then did they have?» (Euphrosyne 18, 1990, S. 89). pinnas ... non suas bedeutet "Flügel, die ihnen nicht von Natur aus eigen waren", d.h. "künstliche Flügel"5. Der Gedankengang des überlieferten Textes ist somit untadelig. Der unterschiedliche Ausgang des Flugunternehmens von Vater und Sohn lag an der jeweils gewählten Flughöhe (v. 23) und nicht an den Flügeln, denn beide hatten sie ja keine natürlichen Flügel (sc. so daß der eine vielleicht über kräftigere verfügt hätte als der andere), sondern künstliche (v. 24).

IV 1,7f. cantet et innitens limosae seruus (trad. pronus) harenae, aduerso tardam qui trahit amne ratem.

Die Konjektur seruus ist banalisierend. Daß es sich bei dem qui (v. 8) um einen Knecht handelt, versteht sich von selbst. Dieser müht sich ab, vornübergebeugt, das Schiff gegen die Strömung zu ziehen, und findet auf dem morastigen Treidelpfad nur mühsam Halt. Das überlieferte pronus verleiht dem

\footnotetext{
$5 \quad$ Vgl. OLD s.u. suus B 8a. Für Ovid sind folgende Parallelen zu nennen: Epist. 14.90 noua uacca (i. Io) ... cornua ... in patriis non sua uidit aquis; Met. III 203 (vom verwandelten Actaeon) lacrimaeque per ora / non sua fluxerunt.
} 
Bild Anschaulichkeit und ist neben innitens harenae entgegen Halls Auffassung $^{6}$ nicht mehr und nicht weniger überflüssig als an einer ganzen Reihe von Stellen aus Vergils Aeneis neben Verben wie pendere, incumbere o.ä.: vgl. z.B. Aen. VIII 236 (vom Felsverschluß der Höhle des Cacus) prona iugo laeuum incumbebat ad amnem; X 586 Lucagus ut pronus pendens in uerbera telo / admonuit biiugos; XI 484f. frange manu telum Phrygii praedonis et ipsum / pronum sterne solo ${ }^{7}$.

Bei der Begutachtung der Gefangenen im Triumphzug:

IV 2,35f. illo, qui sequitur, dicunt mactata ministro saepe requirenti (trad. recusanti) corpora capta deo.

Die Konjektur requirenti zerstört die Pointe der Stelle. Ovid läßt hier durch ein einziges Wort, unerwartet eingebaut in den Zuschauerkommentar zu einem der vorbeiziehenden Gefangenen, den zivilisierten Römer erklären, daß Menschenopfer keinem Gotte wohlgefällig seien ${ }^{8}$. Der dumme Barbar wähnte, sich seinen Gott durch das kultische Abschlachten gefangener Feinde (mactata saepe corpora capta deo) gnädig stimmen zu können. Daß er nun gefesselt im Triumphzug mitgeführt wird, ist der sinnfällige Beweis für den gegenteiligen Effekt: der Gott hat sich von ihm abgewandt, weil er diese Art von Opfer verschmäht - recusanti!

IV 6,25f. tristior est etiam praesens aerumna priore: ne ( $\mathrm{J} ; u t$ cett. codd.) sit enim sibi par, creuit et aucta mora est.

Es besteht kein Grund, in v. 26 mit ne die singuläre Lesart einer textkritisch irrelevanten Handschrift zu übernehmen. Konzessiv gefaßt, ergibt $u t$ einen einwandfreien Sinn: Nachdem der Betroffene die aerumna, die Strafe in ihrer Grausamkeit, erst einmal kennengelernt hat, kann er sich ausmalen, wie es weitergeht. Deswegen wird sie, wenn auch objektiv gesehen die gleiche bleibend (ut sit enim sibi par), für das subjektive Empfinden immer unerträglicher, je länger sie währt (creuit et aucta mora est) ${ }^{9}$. Zu diesem Gedanken vgl. v. $37 \mathrm{f}$.

6 LCM 16, 1991, S. 83: «it adds absolutely nothing to the sense».

Vgl. auch Aen. I 115f., V 147, V 332f. und IX 712f.

$8 \mathrm{Zu}$ diesem Gedanken vgl. Trist. IV 4.81 (im Zusammenhang mit der Opferung Iphigenies) deae ... crudelia sacra perosae.

9 So versteht schon V. Loers diese Stelle in seiner adnotierten Edition der Tristien (Trier, 1839). 
tulimus patientius ante / quae mala sunt longo multiplicata die und Pont. 1,2,38 et grauior longa fit mea poena mora.

IV 6,33f. integer est melior nitidis gladiator in armis, quam cui tela fero (trad. suo) sanguine tincta rubent.

Das überlieferte suo ist hier unabdingbar. Es geht um den Gegensatz zwischen dem unversehrten (v. 33) und dem bereits angeschlagenen Gladiator (v. 34). Die Verwundung des letzteren bringt Ovid nicht durch ein konträr korrespondierendes Adjektiv zu integer, wie etwa saucius, zum Ausdruck, vielmehr arbeitet er sie subtil ein in die Beschreibung des Äußeren der beiden Kämpfer: der eine hat eine glänzende Rüstung, er ist noch frisch und unverwundet; die Waffen seines Kontrahenten sind - offensichtlich infolge eines vorangegangenen Kampfes - rot, und nicht etwa rot vom Blut des Feindes, sondern von seinem eigenen: tela suo sanguine tincta rubent!

IV 9,7 flagrant odio tua pectora nostri (trad. nostro)

Überliefert und zu halten ist nostro. Die Verwendung des Possessivpronomens anstelle des Genetivus obiectivus des Personalpronomens ist in der lateinischen Literatur keine ungewöhnliche Erscheinung ${ }^{10}$. Aus der augusteischen Dichtung nenne ich nur folgende Stellen: Verg., Ecl. 8.81 nostro Daphnis amore liquescit; Hor., Epod. 5.81 amore sic meo flagres; Prop. IV 3.28 opto / e desiderio sit color iste meo; Ov., Met. II 293 nec fratris nec te mea gratia tangit.

V 13,29f. sic ferat ac referat tacitas nunc littera uoces, et peragat (HP; peragant cett. codd.) linguae charta manusque uices.

Offenbar faßt Hall manusque mit Heyworth als Genetiv auf, der linguae koordiniert ist ${ }^{11}$. M.E. ist manusque Nominativ Plural, zusammen mit charta also Subjekt, und verlangt als Prädikat die wesentlich besser überlieferte Verbform peragant. Es liegt hier eine interpretatio vor: littera ferat ac referat tacitas uoces $\sim$ charta manusque peragant uices linguae - der Brief (littera),

10 Vgl. R. Kühner / C. Stegmann, Ausführliche Grammatik der lateinischen Sprache, Zweiter Teil: Satzlehre, Bd. 1, Hannover, $1912^{2}$ (Ndr. Darmstadt, 1971), S. 599 A. 5; J. B. Hofmann / A. Szantyr, Lateinische Syntax und Stilistik, München, 1965, S. 66.

11 S. J. Heyworth, PCPhS 41, 1995, S. 144, glaubt, daß Ovid mit manusque uices auf eine Funktion des Briefs als Ersatz für «embrace (or gesture)» abhebe. 
mit anderen Worten Papier und schreibende Hand (charta manusque), müssen die gesprochene Rede (uoces bzw. lingua) ersetzen.

\section{II}

Besonders zahlreich sind im neuen Text der Tristien Herstellungen und Beseitigungen von Wortwiederholungen, die der Herausgeber entsprechend seinen Vorstellungen von den stilistischen Prinzipien Ovids vornimmt, vorzugsweise gegen die gesamte Überlieferung.

Zur Rechtfertigung arbiträrer Entscheidungen dienen Begründungen wie: «After iamne in the hexameter, it would, I believe, be a considerable stylistic improvement to have iamne again in the pentameter» (Euphrosyne 16, 1988, S. 134 zu III 12.52) ${ }^{12}$ oder: «To the two instances of tempora here in consecutive lines \{sc. V 10.11f. \} must be added a third, in line 5; and that is too much» (Euphrosyne 18, 1990, S. 96) ${ }^{13}$. Stört Hall sich im letztzitierten Fall an dreimaligem tempora innerhalb von acht Versen, so erscheint in seiner eigenen Textgestaltung von III 8,14-20, d.h. innerhalb von sieben Versen, viermal precari - «That would be very much in Ovid's manner» (LCM 16, 1991, S. 83). Überliefert sind dort jeweils zweimal precari (v. 14 und 17) und rogare (v. 17 und 20). Eingriffe dieser Art sind als methodisch unzulässig zurückzuweisen, weil hier Ovids Stil unreflektiert mit dem eigenen, subjektiven stilistischen Empfinden ineinsgesetzt wird. Zur Definition von Ovids Stil müßte erst einmal im Sinne einer positivistischen Absicherung das Material für die einzelnen Stilistika gesammelt, vor allem aber müßte untersucht werden, ob bzw. inwieweit Ovid denn überhaupt dazu neigt, ein bestimmtes Stilmittel in bestimmten Situationen und Kontexten mehr oder weniger stereotyp einzu-

12 Vgl. auch Halls Ausführungen zu seiner Fassung von II 555f. coeptaque (trad. dictaque) sunt nobis, quamuis manus ultima coeptis / defuit, in facies corpora uersa nouas: «I can see absolutely no reason why Ovid should have begun line 555 with dicta and ended it with coeptis» (Euphrosyne 16, 1988, S. 130). Mit dieser Begründung «beseitigt \{Hall\} ein entscheidendes Selbstzeugnis über die Arbeit an den Met., die demnach im J. 9 erst begonnen, nicht bereits verfaßt (dicta) gewesen wären» (Häuptli \{wie Anm. 1\}, S. 326).

13 Vgl. auch Aussagen wie: «The repetition of ... strikes me as pointless and not very elegant» (Euphrosyne 18, 1990, S. 87); «Altogether more pointed would be the phrase ...» (Euphrosyne 16, 1988, S. 126); «More vigorous and more precise, I suggest, would be ...» (Euphrosyne 16, 1988, S. 135); «A small change, but idiomatic, and Ovidian» (Euphrosyne 20, 1992, S. 141). 
setzen. Allein wenn sich zeigen ließe, daß Ovid seinen stilistischen Schmuck in stereotyper Weise und damit berechenbar anwendet - zu diesem Ergebnis aber wird man gerade bei diesem Dichter schwerlich gelangen können -, wären Textänderungen zu rechtfertigen, die allein oder in erster Linie auf stilistischen Argumenten basieren.

Auf einige Fälle von Texteingriffen aus stilistischen Gründen möchte ich im folgenden genauer eingehen:

I 5,81f. (= I 5b,37f.) denique quaesitos tetigit tandem (trad. tamen) ille Penates, quaeque diu petiit, contigit arua tamen.

tandem, eine von Wassenbergh übernommene Konjektur, zerstört die stilistische Pointe des Distichons. Ovid doppelt hier im Sinne einer interpretatio die Aussage, daß Odysseus nach großen Leiden die Heimkehr erleben durfte. In diesem Rahmen unterstreicht er durch das zweifache tamen in nachdrücklicher Weise, daß der Fall jenes berühmten Dulders längst nicht so entsetzlich war wie sein eigener. Bei jenem gab es zuletzt trotz allem noch ein 'dennoch!', bei unserem unglücklichen Dichter gibt es dies nicht: v. 83 (= I 5b.39) at mihi eqs.

\section{5-8 carmina fecerunt ut me cognoscere uellent omine non fausto femina uirque mihi (trad. meo); carmina fecerunt uitam ut (trad. ut me) moresque notaret iam demi iussa Caesar ab Arte meos.}

Es besteht kein Anlaß daran zu zweifeln, daß Ovid diese beiden zentralen Distichen des kunstvoll als Ringkomposition gestalteten Beginns des zweiten Tristienbuchs, v. 1-12, willentlich parallel gebaut hat. Der den ganzen Abschnitt bestimmende Gegensatz von carmina und ego wird hier in seiner Ambivalenz zum Ausdruck gebracht: die beiden Elemente stehen einerseits als Gegenpole zweimal nacheinander in der innerhalb eines Distichons größtmöglichen Entfernung (v. 5f. Carmina ... / ... meo ${ }^{14} /$, v. 7f. Carmina ... / ... meos /), andererseits wird ihre Untrennbarkeit dadurch sprachlich abgebildet, daß meo und carmina als Schlußwort des ersten und Anfangswort des zweiten

\footnotetext{
14 Nicht mihi. Das Possessivpronomen meus spielt eine nicht unerhebliche Rolle in der stilistischen Gestaltung des ringkomponierten Abschnitts II 1-12, nimmt es doch insgesamt viermal die betonte Schlußposition eines Distichons ein: im ersten, im letzten und in den beiden mittleren. Der Eingriff Halls, der diese Komposition empfindlich stört, müßte sich auf sehr gravierende Argumente stützen können. Diese wurden bisher nicht vorgebracht, auch nicht in der m.W. jüngsten Erörterung der Stelle durch S. J. Heyworth (PCPhS 41, 1995, S. 140).
} 
Distichons unmittelbar zusammentreffen und gemeinsam genau die Mitte von 2,1-12 einnehmen. In diesem Zusammenhang dient die ausgeprägte, vier Wörter umfassende Anapher in v. 5 und 7, carmina fecerunt ut me, der emphatischen Unterstreichung der Aussage des ersten und letzten Distichons des Abschnitts (v. 1f. und 11f.), wo der Dichter über die verhängnisvollen Auswirkungen der Dichtung auf sein Leben klagt: die Gedichte allein - hier werden sie zum handelnden Subjekt! - bewirkten Ovids Elend. Halls Veränderung von ut me moresque notaret $\mathrm{zu}$ uitam ut moresque notaret in $\mathrm{v} .7$ beseitigt einen Teil der Anapher um eines nicht erkennbaren Fortschritts willen ${ }^{15}$. Ein vergleichbarer Fall von stilistischer Gestaltung, die der starken Emphase dient, ist der Anfang von Elegie 5,6. Auch hier ist Halls Eingriff, der den äußeren Ring einer doppelten Anapher (v. 1 und 3 tu quoque, v. 2a und 2b qui mihi) beseitigt, inakzeptabel:

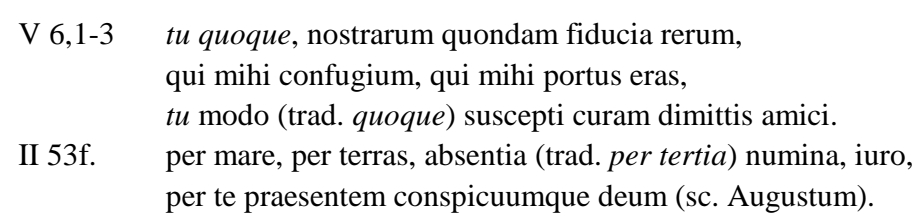

Die Überlieferung ist einwandfrei zu verstehen. Die tertia numina sind die Gottheiten der Unterwelt, des dritten Bereichs neben Oberwelt und Meer. Halls Konjektur stellt hier, offenbar inspiriert von V 2,45 (= V 2b,1) adloquor en absens absentia numina supplex, die pointierte Antithese absentia numina praesens deus her. Gerade das «Nebeneinander von An- und Abwesenheit» (Wieland), ist der Rede- wie der Denkweise Ovids natürlich keineswegs fremd $^{16}$, aber daraus allein läßt sich nicht für eine bestimmte Stelle die Berechtigung eines konjekturalen Eingriffs in eine sinnvolle Überlieferung ableiten.

15 Hall stört sich daran, daß me neben mores ... meos tautologisch sei (vgl. Euphrosyne 18, 1990, S. 88). Diesem Mißstand, der ohnehin nur dann besteht, wenn man an poetische Diktion sprachökonomische Gesichtspunkte heranträgt, die dieser wesensfremd sind, schafft die vorgenommene Änderung von me zu uitam ganz gewiß keine Abhilfe. Eine Parallele zu der vorliegenden Stelle ist IV 10,105 oblitusque mei ductaeque ... uitae. Auch hier erkennt Hall auf Tautologie und ändert mei zu aeui. Wiederum erscheint der Eingriff wenig geeignet, den Anstoß zu beseitigen.

16 Vgl. H. Wieland, «Musa mea est index. Redeweise als Ausdruck der Denkweise bei Ovid», WSt 109, 1996, S. 99-117, hier bes. S. 105ff. 
III 11,39-42 saeuior es tristi Busiride, saeuior illo, qui falsum lento torruit igne bouem, et tostum (trad. quique bouem) Siculo fertur donasse tyranno, et dictis artes conciliasse suas.

In v. 40f. stellt Hall eine Epiploke her (torruit ... et tostum), eine Stilfigur, die Ovid zweifelsohne liebt (s.u.). Die Willkür dieser Konjektur befremdet um so mehr, als sie dem Kriterium des Sinns keine Rechnung trägt. Der Tyrann Phalaris erhält schließlich keinen gerösteten Stier als Geschenk, sondern ein Folterinstrument. In den zitierten Versen handelt es sich zunächst (v. 39f.) um eine periphrastische Antonomasie der Person des berühmt-berüchtigten Perillus durch die Beschreibung seiner Erfindung. Der Name fällt auch im weiteren Kontext nicht. Perillus hatte einen Stier aus Metall konstruiert, der mit einer Befeuerungsanlage ausgestattet war und innen einen Hohlraum bot. Daß torruit (v. 40) sich weniger auf den Stier selbst bezieht als auf den Delinquenten, dem der Platz in seinem Innern zugewiesen würde ${ }^{17}$, ist die Pointe, die der Dichter unausgesprochen läßt, um dem lector doctus die intellektuelle Freude des Verstehens $\mathrm{zu}$ bereiten. Mit quique (v. 41) leitet Ovid dann von der umschreibenden Einführung des Hauptakteurs über zu der eigentlichen, als warnendes Exemplum für seinen Gegner gedachten Erzählung, wie jener durch v. 39 als saevus charakterisierte Unmensch sein verdientes Ende fand (v. 41$54)^{18}$.

Zum Schluß soll noch eine Stelle erörtert werden, an der die Überlieferung seit Madvig bereits mehrfach angezweifelt wurde:

I 3,99 et uoluisse mori, moriendo ponere sensus.

Hall ändert mit Luck den Text zu et uoluisse mali moriendo ponere sensum, offenbar mit Blick auf Pont. I2,30, wo es von Niobe heißt: quae posuit sensum saxea facta mali $^{19}$. Zugegebenermaßen ist dies verlockend. Jedoch sollte der

17 Vgl. Ars I 653f. et Phalaris tauro uiolenti membra Perilli / torruit und Pont. II 9,44 quiue (i. Phalaris) repertorem torruit arte sua.

18 Nicht berechtigt ist daher Halls Kritik: «... the sequence qui ... bovem, quique bovem, where qui ... qui refers to one and the same individual, Perillus, and the double anaphora is totally devoid of point, a sheer waste of space». (Euphrosyne 20, 1992, S. 143).

19 Keiner von beiden beruft sich allerdings expressis uerbis auf diese Stelle. Für mali führt Hall einen textkritisch irrelevanten Vaticanus (V6) an. Die Fügung mali sensum findet sich auch Trist. IV 6,22, vgl. außerdem Pont. IV 16,50 sensum materiamque mali. 
Umstand, daß Ovid gerade in der Exildichtung so manchen seiner Gedanken zwar bis zum Überdruss wiederholt, sprachlich aber immer wieder in neuem Gewande präsentiert, davor warnen, beim Vorliegen einer inhaltlichen Parallele so ohne weiteres auch die gleiche sprachliche Gestaltung zu erwarten. Zunächst stellt sich die Frage, ob es denn notwendig ist, den überlieferten Text anzutasten. Argwohn erregte neben der Wendung moriendo ponere sensus vor allem die Juxtaposition von mori und moriend $o^{20}$. Letztere verteidigte jedoch zu Recht bereits Lenz gegen Luck als «eine echt ovidische Stilisierung» (Gnomon 40, 1968, S. 472). Zur Untermauerung dieses Urteils seien folgende Stellen aus Ovids Tristien angeführt, sowohl Belege für die Figur der Epiploke als auch weniger strenge Typen von unmittelbarem Nebeneinander zweier Formen desselben Verbs ${ }^{21}$ : I 1,38 tempora rerum / quaerere; quaesito tempore tutus eris; I 8,7 omnia iam fient, fieri quae posse negabam; I 9,26 cum quis in aduersis, siquid amauit, amat; II 21 Musaque, quam mouit, motam quoque leniat iram; II 511 haec tu spectasti spectandaque saepe dedisti; III 9,27 atque ita diuellit diuulsaque membra per agros / dissipat; V 2,18 uulnera qui fecit, facta leuare uelit. Strukturell und thematisch unserer Stelle sehr ähnlich, wenn auch ohne unmittelbare Juxtaposition der beiden Verbformen, ist I 11,24 quam (sc. mortem) dubia timeo mente timensque precor.

Inwiefern die Formulierung moriendo ponere (= deponere) sensus ${ }^{22}$, d.h. "durch den Tod die Wahrnehmungsfähigkeit ablegen", im Hinblick auf einen

20 Vgl. J. N. Madvig, Adversaria critica II, Kopenhagen, 1873, S. 96 («ego neque quid hac geminatione [mori, moriendo p.s.] efficiatur, video, neque usitate dici puto, nulla re adiecta, ponere sensus») und G. Luck, P. Ovidius Naso, Tristia, Bd. II: Kommentar, Heidelberg, 1967, S. 46 zur Stelle.

${ }^{21}$ Fälle von reiner Geminatio sind nicht berücksichtigt. Vgl. aus den übrigen Dichtungen Ovids: (a) Epiploke: Am. III 8,7 cum bene laudauit, laudato ianua clausa est, Epist. 20,73 idem qui facimus, factam tenuabimus iram, Met. I 33 congeriem secuit sectamque eqs., IV 64 tegitur, tectus, X 282f. temptat / temptatum, Pont. I 10,23 sed uigilo uigilantque mei sine fine dolores; ohne unmittelbare Juxtaposition: Trist. III 5,11 uidi ... uultus uisosque notaui, Met. I 402 mollirique ... mollitaque, II 768ff. uidet ... inuidiam uisaque eqs., Fast. III 21 videt ... visamque. (b) sonstige Formen (besonders häufig in den Liebeselegien): Am. I 4,58 quidquid ibi poteris tangere, tange, mei, II 4,39 capiet, capiet, II 11,22 credite! credenti, II 19,48 incipis, incipiet, III 2,59 promisit, promittas, III 3,32 timuere, timent, Ars I 270 posse capi: capies, I 310 si mauis ... fallere, falle, I 503 cum surgit, surges, II 30 si non uis puero parcere, parce seni, u.a.

22 Zur Bezeichnung des Sterbens sind in der Dichtung und in der poetisierenden kaiserzeitlichen Prosa mehrere Junkturen mit ponere als Simplex pro composito gut belegt: animam ponere, uitam ponere, spiritum ponere, lucem ponere (vgl. OLD s.u. ponere $10 \mathrm{~b}$ ). 
sinnvollen Gedankengang der Passage etwas vermissen lassen sollte, vermag ich nicht zu erkennen. Die Seelenlage der Frau Ovids ist durch den Kontext hinreichend klar. Sie möchte sich durch den Tod von ihrem unerträglichen Leid befreien, wovon denn sonst? ${ }^{23}$ Ein Genetivattribut wie mali ist nicht nur unnötig, es zieht sogar, wie Luck (Kommentar zur Stelle) gesehen hat, die Notwendigkeit eines weiteren Eingriffs nach sich, die Änderung von sensus zu sensum. Mit der Junktur ponere sensus beschreibt der Dichter eben jenen Sachverhalt aus der Perspektive des Sterbenden, den Cicero aus der anderen Perspektive, der des Todes, mit der Junktur sensus auferre formulierte: Tusc. I 97 necesse est enim, ut ... sensus omnino omnes mors auferat.

23 Die von J. Diggle («Notes on Ovid’s Tristia, Books I-II», CQ 30, 1980, S. 401-419, hier S. 408f.) aus der griechischen Tragödie angeführten Parallelen für die verbreitete Auffassung vom Tod als dem Erlöser aus Leid sind daher ohne Relevanz für die Frage nach der Notwendigkeit eines Genetivattributs, das den Inhalt der Sinneswahrnehmung beschreibt. 